\title{
INCIDENCIA DEL IMPUESTO A LAS GRANDES TRANSACCIONES FINANCIERAS DE LA EMPRESA PRIVADA VENEZOLANA
}

\author{
Chess Emmanuel Briceño Núñez* \\ Yosmary Carolina Durán Sánchez** \\ Alen David Montilla Soto****
}

\section{DOI: I0.5377/EYA.VI2II.I2963}

Recibido: 05/06/2021 Aceptado: 15/11/2021

\section{RESUMEN}

La presente investigación se basa en realzar las posibles repercusiones económicas que puede llegar a generar el impuesto dentro de la administración del efectivo de la empresa, cuyo estudio se hizo bajo una metodología descriptiva de campo. Tiene como objetivo analizar la incidencia del Impuesto a las Grandes Transacciones Financieras (IGTF) en la administración del efectivo en la empresa privada venezolana, y como caso de estudio se tuvo a la empresa Vinccler C.A. La población está conformada por 02 empleados del área Administrativa-Contable. Para la recolección de datos, se utilizó una guía de entrevista semi estructurada. Una vez obtenida la información requerida, se prosiguió al procesamiento de los resultados, los cuales evidenciaron que la empresa actualmente implementa la técnica del presupuesto de efectivo, de esa manera organiza su dinero y cumple con el pago del IGTF, además, Vinccler cumple con los respectivos deberes formales que establece la Ley del IGTF y de ese modo evita las sanciones, cierres y multas por parte del SENIAT. Finalmente, también se pudo conocer que la empresa tiene pleno conocimiento de los elementos que los conducen a cumplir con el pago del impuesto y toman sus medidas para evitar las erogaciones de dinero innecesarias que obstruyan la administración eficiente del efectivo.

PALABRAS CLAVES: Administración del efectivo, deberes formales, Impuesto a las grandes transacciones financieras.

JEL: H20

\footnotetext{
* Profesor universitario e investigador, Universidad Nacional Abierta de Venezuela. https://orcid.org/0000-0002-1712-4136 Correo electrónico: chesspiare@gmail.com Caracas, Distrito Capital, Venezuela.

** Profesor universitario, Universidad de Los Andes de Venezuela. https://orcid.org/0000-0003-1972-07488 Correo electrónico: yosmaryduran@gmail.com Trujillo, Venezuela.

*** Estudiante de maestría, Universidad Nacional Experimental Libertador. https://orcid.org/0000-0001-9765-0672 Correo electrónico: alen. montilla.soto@gmail.com Trujillo, Venezuela.
} 


\title{
INCIDENCE OF THE TAX ON LARGE FINANCIAL TRANSACTIONS OF VENEZUELAN PRIVATE COMPANIES
}

\author{
Chess Emmanuel Briceño Núñez* \\ Yosmary Carolina Durán Sánchez** \\ Alen David Montilla Soto***
}

\section{DOI: I0.5377/EYA.VI2II.I2963}

Recived: 05/06/2021 Accepted: $15 / 11 / 2021$

\begin{abstract}
This research is based on highlighting the possible economic repercussions that the tax may generate within the cash administration of the company, the study of which was carried out under a descriptive field methodology. Its objective is to analyze the incidence of the tax on large companies financial transactions in the administration of cash in the Venezuelan private company, and as a case study we had the company Vinccler CA The population is made up of 02 employees of the Administrative-Accounting area. For data collection, a semi-structured interview guide was used. Once the required information was obtained, the results were processed, which showed that the company currently implements the cash budgeting technique, in this way it organizes its money and complies with the payment of the IGTF, in addition, Vinccler complies with the respective formal duties established by the IGTF Law and thus avoids sanctions, closures and fines by SENIAT. Finally, it was also learned that the company has full knowledge of the elements that lead them to comply with the payment of the tax and take their measures to avoid unnecessary money outlays that obstruct the efficient administration of cash.
\end{abstract}

KEYWORDS: Cash management, formal duties, Tax on large financial transactions.

JEL: H20

\footnotetext{
* University professor and researcher, National Open University of Venezuela. https://orcid.org/0000-0002-1712-4136 Email: chesspiare@ gmail.com Caracas, Distrito Capital, Venezuela.

** College professor, University of the Andes of Venezuela. https://orcid.org/0000-0003-1972-07488 Email: yosmaryduran@gmail.com Trujillo, Venezuela.

*** Masters student, Libertador National Experimental University. https://orcid.org/0000-0001-9765-0672 Email: alen.montilla.soto@gmail. com Trujillo, Venezuela. 


\section{INTRODUCCIÓN}

En Venezuela al igual que en distintos países del mundo existe la obligación de pagar impuestos, esto con la finalidad de que los ciudadanos aporten a la cancelación del gasto público y de cierto modo asuman su responsabilidad de contribuyentes. La tributación cuenta con una serie amplia de impuestos que gravan diversas actividades tales como la renta, el valor agregado, la venta y expendio de bebidas alcohólicas, entre otros, en este caso particular se centró en el Impuesto a las Grandes Transacciones Financieras (IGTF) con la finalidad de conocer el posible impacto económico negativo que genera el ser contribuyente de este tributo y de esa manera enfocar la incidencia que dicho impuesto produce en lo que respecta la administración del efectivo.

Al indagar sobre el IGTF en la Ley y su providencia, resulta interesante desglosar ciertos factores que ayuden a reflejar la posible problemática administrativa que se presenta en una organización sujeta al pago de este impuesto, para llevar a cabo el desarrollo de la investigación se consideraron tres aspectos importantes:

El primero de ellos tiene que ver con las técnicas de la administración del efectivo, la intención es conocer si de algún modo el uso de estas ayuda a mejorar el desenvolvimiento económico de la empresa tomando en cuenta la responsabilidad judicial y monetaria que el pago del nuevo Impuesto a las Grandes Transacciones Financieras genera y de esa manera se cumpla correctamente con el desarrollo de la organización y el objetivo de la empresa (maximizar sus utilidades).

En segundo lugar, se estudiaron los deberes formales establecidos en la ley del Impuesto a las Grandes Transacciones Financieras y, por consiguiente, a los que contiene el Código Orgánico Tributario (COT) debido a que guardan relación directa con el impuesto. Al saber que los deberes formales constituyen obligaciones que deben cumplir los contribuyentes, responsables y terceros, de acuerdo con las disposiciones establecidas en las Leyes Especiales y Reglamentos, es importante conocerlos para evitar de ese modo las sanciones y multas que el incumplimiento de los deberes produce y que por su parte también requiere una salida de dinero.

El tercer factor para considerar, fueron los elementos del cumplimiento del Impuesto a las Grandes Transacciones Financieras que de una u otra forma también afectan las finanzas de la empresa.

\section{Marco conceptual}

\subsection{ConteXtualización Situacional}

En Venezuela existe un Sistema Tributario que está formado y constituido por un conjunto de cargas a los cuales se aplican tres poderes: Nacional, Estadal y Municipal. Teniendo cada uno competencia en diferentes imposiciones y rigiéndose bajo ciertos principios que permitan que su recaudación se ejecute de manera legal y transparente, donde se incluya en forma general a todo el sector de la población para el pago del tributo considerando la capacidad económica del contribuyente, de este modo se implementa la progresividad para garantizar una mejor calidad de vida junto con la no confiscatoriedad que protege el derecho de propiedad.

Teniendo en cuenta que los tributos integran una pieza fundamental en la economía de un país, es importante definirlo; Noya et al. (2014) indican: "los tributos constituyen la principal fuente de recursos con que cuenta el Estado para afrontar los gastos que se originan en la realización de sus fines políticos, económicos y sociales". (p. 98). Es decir, la obligación dineraria establecida por la ley, cuyo importe se destina al sostenimiento de las cargas públicas. 
Para brindar un conocimiento amplio sobre los tributos es importante conocer que existen en tres tipos: las tasas, las contribuciones especiales y los impuestos; cada uno de ellos tiene sus características y por lo tanto se recaudan de forma diferente.

Al momento de definirlos Noya et al. (2014) indican que "la tasa implica un desarrollo de una actividad por parte del Estado, que afecta al obligado en virtud de un beneficio que el Estado le proporciona por una actividad”. (p.123).

Es conveniente resaltar que existen dos tipos de tasas: las judiciales, que pudieran originarse en actuaciones jurídicas al momento de ejecutar juicios civiles o sucesorios, entre otros; y las tasas administrativas, que son las que inician a través de la concepción de un documento, certificado o licencia.

El siguiente punto trata de las contribuciones especiales, Noya et al. (2014) dicen que "son tributos recaudados entre contribuyentes que percibieron y/o perciben un beneficio por parte del Estado”. (p. 121), se conocen las contribuciones parafiscales y las contribuciones de mejoras.

Para finalizar con la clasificación de los tributos y definir uno de los principales aspectos de la investigación, Noya et al. (2014) señala que "los impuestos recaen sobre cada contribuyente, sin tener en cuenta los beneficios individuales recibidos por la provisión de los bienes públicos financiados con los mismos”. (p. 119).

En resumen, las personas están obligadas a pagar distintos tipos de tributos por medio de la administración pública, esta imposición se sustenta por la Constitución de la República Bolivariana de Venezuela (1999) en su artículo 133 donde se establece que: Toda persona tiene el deber de coadyuvar a los gastos públicos mediante el pago de impuestos, tasas y contribuciones que establezca la ley.

Dicho esto, el pago se puede ejercer en diferentes circunstancias y periodos de tiempo, indistintamente porque sean periódicos debido a que se genere una renta o instantáneos y se haga de manera inmediata. El tributo consiste generalmente en un recurso de carácter monetario, puesto que su objetivo es la financiación de los gastos públicos.

A modo de realzar el alcance de los tributos, específicamente de los impuestos, se toma como propósito principal de la investigación estudiar el Impuesto a las Grandes Transacciones Financieras (IGTF) cuya ley entró en vigencia el 1o de febrero del año 2016. De acuerdo con la exposición de motivos de esta Ley se puede concretar que el impuesto recae únicamente sobre las transacciones financieras efectuadas por personas jurídicas y las entidades económicas sin personalidad jurídica, calificadas como sujetos pasivos especiales por el Servicio Nacional Integrado de Administración Aduanera y Tributaria (SENIAT), dejando libre del gravamen al resto de las personas jurídicas, así como a las personas naturales.

Entendiéndose como persona jurídica, definido en el Diccionario Básico Tributario Contable (2016), como "el ente ficticio, capaz de ejercer derechos y de contraer obligaciones, además de contraer obligaciones civiles y de ser representada judicial y extrajudicialmente" (S/P). De acuerdo con el concepto anterior, se toman como ejemplo de personas jurídicas: el Estado, las sociedades mercantiles, sociedades civiles entre otras.

En cuanto al proceso de la recaudación del Impuesto a las Grandes Transacciones Financieras, está determinado

(7)Una incubadora de empresas es "un ambiente controlado que nutre la creación, crecimiento y protección de una nueva empresa, en una etapa temprana, antes de que se encuentre lista para operar de manera auto sostenible por sus propios medios. El término ambiente controlado abarca tanto elementos físicos, como virtuales" Gutiérrez (2008). 
esencialmente por 7 aspectos: hechos imponibles u operaciones a las que se aplica, las exenciones, alícuota y base imponible, agentes de retención, la forma de declaración y pago, plazo de pago y los deberes formales.

E1 Impuesto a las Grandes Transacciones Financieras fue creado con la finalidad de gravar cada transacción monetaria que ejerza o involucre al contribuyente especial, aplicándose una alícuota del 0,75\% al monto reflejado en la transacción. Sin embargo, es importante recordar que en la Ley que rige este tributo se encuentran ciertos movimientos mercantiles y entidades financieras que se encuentran fuera de este gravamen y por tanto son consideradas como exentas del pago. Resulta pertinente resaltar que en Venezuela un contribuyente especial son los sujetos que han sido expresamente calificados y notificados por el Servicio como tales. Atienden al nivel de ingresos brutos anuales, según la Gerencia de Tributos Internos de la Región de su jurisdicción. Estos sujetos pueden ser entes públicos, empresas privadas, personas naturales o jurídicas. Puede que sean contribuyentes ordinarios del Impuesto al Valor Agregado, lo cual dependerá del hecho de que estén o no sujetos al Impuesto y que realicen o no los hechos imponibles según la Ley.

Como una medida para proporcionar información a los contribuyentes y responsables, puesto que es esencial que estos manejen suficiente información en cuanto al proceso de la recaudación del impuesto, la Administración Tributaria creó, además de la Ley del Impuesto a las Grandes Transacciones Financieras y la Providencia Administrativa (SNAT/2016/0005), un portal electrónico en la página web del Servicio Nacional Integrado de Administración Aduanera y Tributarias (SENIAT). En este portal el interesado puede emplear un instructivo de usuario que constituye una herramienta de consulta y apoyo diseñado para la eficiente declaración y pago del Impuesto a las Grandes Transacciones Financieras de acuerdo con lo establecido en la ley.

En proporción con lo anterior y destacando el vínculo que tiene la aplicación y el pago del Impuesto a las Grandes Transacciones Financieras con el patrimonio de los sujetos pasivos especiales, resulta elemental definir y destacar la importancia que tiene el efectivo sobre el impuesto, debido a que es un punto focal en la información que se está manejando; al respecto, Rodríguez (2004) considera que "el efectivo es todo el dinero que tenemos disponible para gastarlo en un momento determinado, tal como monedas, billetes, cheques, depósitos a la vista y otros". (p. 17).

La administración del efectivo permite conocer entre otras cosas el efectivo mínimo de operación y el costo de oportunidad, Block y Hirt (2008) opinan que "la minimización de los saldos de efectivo y saber con precisión las fechas en que se desplaza hacia adentro y hacia afuera de la compañía mejora la rentabilidad general de la corporación". (p. 186).

Con base a la importancia que tiene la implementación del Impuesto a las Grandes Transacciones Financieras y las posibles repercusiones monetarias que genere el cumplimiento de esta obligación en cuanto a la magnitud de los diversos hechos imponibles, se toma como contexto de investigación la empresa Venezolana de Inversiones y Construcciones Clerico, C.A. (VINCCLER, C.A.), específicamente la Oficina Principal de Occidente ubicada en Valera Estado Trujillo, la cual es una contratista general que participa en la Industria de la Construcción desde hace más de 40 años y ejecuta obras de variada naturaleza. Sus servicios integrales abarcan las siguientes especialidades: obras civiles, mecánicas y eléctricas.

A raíz de unavisita a la empresa Vinccler C.A, y por medio de una entrevista informal aplicada ala Administradora, se pudo conocer que dicha unidad económica pertenece a la categoría de Sujetos Pasivos Especiales de acuerdo a la providencia No 0685; por lo tanto, están obligados a pagar el 0,75\% de cada transacción monetaria realizada como aplicación del Impuesto a las Grandes Transacciones Financieras y por consiguiente se detectó 
que existe una deficiencia en el manejo y disposición del efectivo debido a la disminución inmediata del activo disponible en las cuentas a consecuencia del pago del tributo, demostrando así, que no operan un control del uso de efectivo que les permita saber con precisión y antelación los saldos totales.

Es importante saber que antes de efectuarse la transacción mercantil dispuesta por la empresa, primero se realiza el cobro del impuesto. En dado caso de que la operación sea exacta al monto total disponible en la cuenta, no se podrá realizar el desembolso deseado, ocasionando la minuciosa supervisión de las cuentas bancarias para garantizar la disposición suficiente de dinero que cubra la transacción y el descuento por cancelación del impuesto. Adicional a esto, las empresas deben mantener dinero para reducir su riesgo de insolvencia técnica, teniendo de esta manera un conjunto de recursos líquidos para realizar tanto los desembolsos planeados como los imprevistos.

El hecho de habilitar cuentas con dinero inactivo desde el punto de vista rentable trae como consecuencia el costo de oportunidad, esto es, el capital que deja de invertir la empresa para generar beneficios económicos. Además de ello, en una visión a corto plazo, es posible un decremento en la utilidad debido a la repercusión que tiene el tributo en la parte financiera. Eyrl impuesto grava, entre otras cosas, las entradas y salidas de dinero, dicho de otra manera, los importes que corresponden al pago del impuesto vienen dados por la mayoría de las operaciones mercantiles que realiza la compañía, disminuyendo esto la proporción de las ganancias.

Por otra parte, la aplicación del Impuesto a las Grandes Transacciones Financieras produce un efecto encarecedor debido a que la empresa Vinccler C.A se ve en la necesidad de incrementar el costo del servicio, tomando en cuenta que este tributo se califica contablemente como un gasto, la entidad debe de alguna manera tratar de respaldar esta erogación y a su vez esos ingresos sirven como una medida para suplementar el flujo de caja. Evidenciando que debido a su actividad económica el ciclo del efectivo no se desarrolla de manera general como en las empresas que comercializan productos, puesto a que no manejan un inventario que les permita vender la mercancía ya sea a crédito o al contado, obtener una ganancia y que el efectivo de inversión retorne nuevamente.

Considerando la finalidad de la compañía, los ingresos se generan al momento de establecer una inicial para las obras y el resto de manera proporcional a la ejecución. Esto a su vez, conlleva a disponer de una adecuada administración en cuanto los desembolsos totales para la realización de trabajos, con el propósito de minimizar las variaciones de precios en los elementos del costo, entendiéndose estos como los materiales directos, mano de obra directa y los costos indirectos de fabricación y de este modo evitar posteriores salidas de dinero adicionales considerando la situación del país y la fluctuación de la economía.

En este orden de ideas, la Empresa Vinccler C.A debería darle importancia a la implementación de medidas administrativas que le conceda una programación eficiente de los fondos a utilizar, haciendo uso de una planificación de administración del efectivo y un plan de compras, como un instrumento que le permita coordinar financieramente sus activos.

\subsection{IMPUESTOS}

Este tributo constituye uno de los principales ingresos del país para financiar el gasto social, así como bienes y servicios públicos, para Otálora (2009) "es un pago efectuado al Estado por mandato de la ley, en el que no existe una retribución específica en servicios públicos o bienes por el valor equivalente al monto pagado”. (p. 132).

Así mismo, Noya et al. (2014) dividen dos tipos de impuestos, los directos "son aquellos que gravan manifestaciones inmediatas de capacidad contributiva, como la ganancia neta o el patrimonio". (p. 119), y los 
indirectos considerados "aquellos que gravan manifestaciones mediatas de capacidad contributiva, como el consumo". (p. 120).

En los impuestos directos se toman en consideración las circunstancias personales que influyen en la capacidad económica del contribuyente, tal como la imposición progresiva sobre la renta global que tienen en cuenta situaciones como las cargas de familia, el origen de renta y otras circunstancias, mientras que en los impuestos indirectos se vincula con actos o situaciones accidentales.

\subsection{Contribuciones}

Es la clase de tributo cuya base para gravarlo es la obtención por el contribuyente de un beneficio o de un aumento de valor de sus bienes como consecuencia de la realización de obras públicas, Noya et al. (2014) dicen que "las contribuciones son tributos recaudados entre contribuyentes que percibieron y/o perciben un beneficio por parte del Estado. La recaudación está en función de los beneficios recibidos; por ejemplo por algunas obras públicas". (p.121). Otálora (2009) además de darle un significado a las contribuciones también las divide en dos tipos:

En el caso de las contribuciones, tenemos las contribuciones a la seguridad social y las contribuciones de mejora. Las primeras, al igual que cualquier tributo, constituyen un pago efectuado por mandato de la ley, con la diferencia de que en este caso existe retribución específica en el momento que el contribuyente solicita la prestación efectiva del servicio. En cambio, las contribuciones de mejoras tienen por objeto recuperar las economías externas que se producen como consecuencia de la realización deunaobrapúblicayquefluyenhaciaungrupoindividualizadodepersonasquesebeneficiandeellas.(p.133)

Ya definida y especificada la clasificación de los tributos como parte importante de la investigación, el siguiente apartado se abocará a darle desarrollo a uno de los impuestos que forma parte de los tributos como lo es el Impuesto a las Grandes Transacciones Financieras junto con las implicaciones de la administración del efectivo que se ven afectadas por el cumplimiento de este tributo dentro de la empresa Vinccler C.A.

\subsection{IMPUESTO A LAS GRANDES TRANSACCIONES FINANCIERAS}

Este gravamen se rige por la ley que establece el Impuesto a las Grandes Transacciones Financieras con Decreto No 2.169 del 30 de diciembre de 2015, publicada en Gaceta Oficial de la República Bolivariana de Venezuela No 6.210 Extraordinario que entró en vigencia el primero (1ro) de febrero de 2016. De acuerdo con la exposición de motivos de la Ley de Impuestos a las Grandes Transacciones Financieras (2015) se puntualiza:

El impuesto propuesto se distingue de otros tributos, de similar naturaleza editados en el pasado, por cuanto recae únicamente sobre las transacciones financieras efectuadas por las personas jurídicas y entidades económicas sin personalidad jurídica, calificadas como sujetos pasivos especiales por el Servicio Nacional Integrado de Administración Tributaria (SENIAT), dejando libre del gravamen al resto de las personas jurídicas, así como también a las personas naturales.

El objeto de este impuesto es gravar las grandes transacciones financieras efectuadas por contribuyentes especiales, quienes son designados por el SENIAT basándose en la providencia No 0685 y cuya administración, recaudación, fiscalización y control de este tributo queda bajo competencia del poder público nacional. Cabe destacar que el arancel no es deducible del Impuesto Sobre la Renta y que la base imponible para la aplicación del impuesto estará constituida por el total de cada débito en cuenta u operación gravada, es decir, por el monto completo de cada acto monetario relacionado con el contribuyente especial, en los casos de cheque de gerencia, será la del importe del cheque. 
La alícuota impositiva por la cual se calcula el pago de este tributo es de 0,75\%, y solo podrá ser modificada a modo de disminución por el Ejecutivo Nacional mediante decreto, vale la pena especificar que el monto a pagar de la obligación tributaria será el que resulte de multiplicar la alícuota impositiva por la base imponible.

Es evidente que el Impuesto a las Grandes Transacciones Financieras guarda relación directa con lo que refiere al efectivo, por consiguiente, resulta necesario que los contribuyentes especiales dispongan de una administración del efectivo eficiente que les permita cumplir con sus obligaciones tributarias además de dirigir y conocer con precisión los cobros y desembolsos dentro de la organización.

\section{Metodología}

\subsection{TiPO DE INVESTIGACIÓN}

En este apartado se indica el tipo de investigación según el grado o nivel de profundidad con el cual se llevará a cabo el proyecto, para abordar el siguiente estudio se empleó una investigación de tipo descriptiva, que, según Arias (2006), "consiste en la caracterización de un hecho, fenómeno, individuo o grupo con el fin de establecer su estructura o comportamiento". (p. 24). La presente investigación es del nivel descriptiva bajo un paradigma cuantitativo por cuanto se basa en analizar la incidencia del Impuesto a las Grandes Transacciones Financieras en la administración del efectivo y sus equivalentes de la empresa Vinccler C.A.

\subsection{DisEÑO DE LA INVESTIGACIÓN}

Para Arias (2006) "El diseño de la investigación es la estrategia general que adopta el investigador para responder al problema planteado”. (p. 27), es decir, la manera reconocida de llevar a cabo el estudio. De acuerdo con las características de la presente investigación resulta conveniente aplicar un diseño de campo, que según Arias (2006), "es aquella que consiste en la recolección de datos directamente de los sujetos investigados, o de la realidad donde ocurren los hechos (datos primarios), sin manipular o controlar variable alguna”. (p. 31). De acuerdo con esto, es preciso señalar que la información se recabó de manera personal por medio de una entrevista a los empleados del sector administrativo en la empresa Vinccler C.A.

\subsection{Población y MUeSTRA}

Para llevar a cabo una investigación es fundamental identificar la población puesto que será objeto de estudio, a través de la población es que se puede acceder a la información y de esa manera responder las interrogantes que dieron inicio a la investigación. Arias (2006) indica que una población objetivo "es un conjunto finito o infinito de elementos con características comunes para los cuales serán extensivas las conclusiones de la investigación”. (p. 81).

A modo de especificar y proporcionar detalles en relación con la actual investigación, una población finita definida por Arias (2006) es la "agrupación en la que se conoce la cantidad de unidades que la integran". (p. 82), es decir, un número previamente definido de las unidades de análisis, para el presente estudio se consideró a Vinccler C.A. como la población, en este caso no se aplicó muestreo por cuanto posee la característica de ser un conjunto finito y además de ello es accesible debido a que está plenamente identificada y se conocen los sujetos informantes.

En esta investigación, se les aplicó el instrumento a dos personas del área administrativa las cuales ocupan los cargos de administrador y asistente administrativo, a través de ellos se pudo recabar la información necesaria para poder llegar al análisis y las conclusiones pertinentes. El tipo de muestreo empleado fue el Muestreo por Conveniencia, considerando que es el que mejor se adapta al contexto de los sujetos y la empresa. 


\subsection{TÉCNICAS E INSTRUMENTOS DE RECOLECCIÓN DE DATOS}

Las técnicas de recolección de datos son en general las diferentes maneras de obtener la información que ayudan a desglosar los objetivos planteados, mientras que los instrumentos son los materiales que se emplean para recolectar y plasmar la información al momento de aplicar las técnicas. De acuerdo con las características de la investigación actual, resultó conveniente aplicar como técnica para la recolección de la información una entrevista cuyo instrumento fue una guía de entrevista semi estructurada compuesta por 18 preguntas referentes a los objetivos propuestos.

Arias (2006) expresa que la entrevista "es una técnica basada en un dialogo o conversación "cara a cara”, entre el entrevistador y el entrevistado acerca de un tema previamente determinado, de tal manera que el entrevistador pueda obtener la información requerida" (p. 74

\subsection{TÉCNICA DE ANÁLISIS Y PRESENTACIÓN DE RESULTADOS}

Debido a la naturaleza de la investigación, la información se analizó de manera cualitativa, considerando que se trata de una entrevista abierta; por tanto, se realizó una codificación a través de un proceso mediante el cual se agrupó la información obtenida en categorías que concentran las ideas otorgadas por la población de estudio.

De acuerdo con Álvares (2005) una vez que la información ha sido recolectada, transcrita y ordenada la tarea consiste en intentar darle sentido. Por esta razón al considerar que es una guía de entrevista, no se elaboraron diagramas para representar la información; esta misma característica conduce a trabajar de manera cualitativa, verificando y contrastando las opiniones recopiladas durante la aplicación del instrumento.

\section{ANÁlisis E INTERPRETACión DE LOS RESUltados}

En este aparto se presenta una examinación de las técnicas de administración del efectivo que maneja la empresa Vinccler C.A al año 2020. Obteniendo la siguiente información:

Item 1: ¿Cómo maneja la empresa la determinación del efectivo minimo que requiere para realizar sus actividades financieras y cumplir con la obligación de pagar el IGTF?

\section{Tabla 1. Respuestas del item 1}

\begin{tabular}{l|l}
\multicolumn{2}{c}{ Entrevistado1 } \\
\hline $\begin{array}{l}\text { La empresa según el monto global de las cuentas mantiene un fondo } 2 \\
\text { proporcional al pago del impuesto. }\end{array}$ & $\begin{array}{l}\text { Al principio de la implementación del impuesto no se tomaban medidas como tal } \\
\text { para administrar el efectivo y por tanto surgió la problemática. Después de unos } \\
\text { meses, la empresa comenzó a ejercer un control en cuanto a la administración del } \\
\text { efectivo, y hasta la actualidad estiman las entradas y salidas de dinero con el fin } \\
\text { de calculary establecer un monto global para el pago del IGTF. }\end{array}$ \\
\hline
\end{tabular}

De acuerdo con la respuesta del primer entrevistado, la empresa se puede ver afectada económicamente por el hecho de mantener un fondo sobreestimado de dinero inactivo para cubrir con la obligación del pago del impuesto, esto lo sustenta Van Horne y Wachowicz (2010) "la mayoría de las empresas de negocios establecen un nivel meta para los saldos de efectivo que deben tener. No quieren saldos con exceso de efectivo porque pueden ganar intereses si invierten estos fondos en valores comerciales". (p. 234).

Con respecto a la respuesta del segundo entrevistado, ratificó la problemática que se presentó en la empresa durante el segundo semestre del 2020 con respecto a nueva responsabilidad que acarrea cumplir con el IGTF, 
al mismo tiempo, se describió que solventaron de cierto modo la administración del efectivo puesto que estiman las entradas y salidas de dinero, esto con la finalidad de calcular el 0,75\% del monto global de las transacciones para hacer el apartado correspondiente al pago del impuesto.

La metodología empleada para conocer los movimientos del efectivo es algo similar a la función que cumple el Presupuesto de Efectivo. Tal como indican Van Horne y Wachowicz (2010) "el presupuesto de efectivo simplemente representa una estimación de los flujos de efectivo futuros". (p. 184), a través de esta técnica la empresa puede cumplir satisfactoriamente con la obligación del pago del IGTF y también manejar de manera eficiente los flujos de efectivo futuro.

Ítem 2: ¿Conocen ustedes la manera en que le puede ayudar a la empresa estas técnicas en cuanto a la administración del efectivo y asi cumplir con el pago del IGTF?

Tabla 2. Respuestas del item 2

\begin{tabular}{l|l}
\multicolumn{2}{c}{ Entrevistado1 } \\
$\begin{array}{l}\text { Al no implementar ninguna de las técnicas como tal, desconocen la manera en } \\
\text { que les puede ayudar en la administración del efectivo. }\end{array}$ & $\begin{array}{l}\text { La empresa implementa una medida parecida a lo que respecta la técnica del } \\
\text { presupuesto de efectivo, y de cierto modo se conocen sus beneficios en cuanto a } \\
\text { la administración del efectivo se refiere para cumplir con el pago del IGTF. }\end{array}$ \\
\hline
\end{tabular}

De acuerdo con el personal entrevistado ambas respuestas difieren, sin embargo, la persona número 2 sí conoce la manera en que le ayuda a la empresa la técnica similar al Presupuesto de Efectivo; pues precisamente a través de la información que este le suministra toman las medidas necesarias, tal como lo describen Van Horne y Wachowicz (2010) "con esta información, el director financiero está en mejor posición para determinar las necesidades futuras de efectivo de la empresa, planear el financiamiento de estas necesidades y ejercer control sobre el efectivo y la liquidez de la empresa”. (p.180).

Ítem 3: ¿Cuál (es) de las técnicas actualmente aplicadas considera usted conveniente para ejercer una adecuada administración del efectivo tomando en cuenta el cumplimiento del IGTF? Y $\dot{\dot{\zeta}}$ Por qué?

Tabla 3. Respuestas del item 3

\begin{tabular}{l|l}
\multicolumn{2}{c}{ Entrevistado1 1} \\
$\begin{array}{l}\text { No implementan ninguna técnica debidamente aprobada por autores, solo } \\
\text { estiman un fondo para el pago del impuesto y para la caja chica. }\end{array}$ & $\begin{array}{l}\text { La técnica antes mencionada ha proporcionado a la empresa un desenvolvimiento } \\
\text { adecuado en cuanto a la administración del efectivo y el cumplimiento del IGTF. }\end{array}$ \\
\hline
\end{tabular}

Con respecto a lo encontrado, existe discrepancia en las respuestas, sin embargo, el segundo entrevistado asegura que la empresa considera que la medida actualmente empleada para administrar el efectivo les ha dado resultados satisfactorios para desenvolverse en el mercado.

De acuerdo con la similitud que tienen la medida adoptada por la empresa Vinccler y la técnica del Presupuesto de Efectivo, Van Horne y Wachowicz (2010) expresan los resultados que arroja el implementar esa técnica para 
administrar su efectivo, puesto que, "nos dice cuánto efectivo es probable que tengamos, cuánto es posible que lo tengamos y por cuánto tiempo”. (p. 222). De esa manera el Presupuesto del Efectivo sirve de apoyo para ejercer control sobre el efectivo y simultáneamente estimar las erogaciones que produce cumplir con el pago del IGTF.

Item 4: ¿̇Han tenido algún inconveniente con el IGTF al momento de implementar alguna técnica para la administración del efectivo? Justifique su respuesta.

Tabla 4. Respuestas del item 4

\begin{tabular}{l|l}
\multicolumn{1}{c|}{ Entrevistado1 } & \multicolumn{1}{c}{ Entrevistado2 } \\
\hline $\begin{array}{l}\text { El fondo estimado para el IGTF es un dinero que no produce rentabilidad y por lo tanto } \\
\text { no genera ningún tipo de ganancias a la empresa. }\end{array}$ & $\begin{array}{l}\text { El dinero que se destina para el pago del IGTF no es productivo y no beneficia a la } \\
\text { organización. }\end{array}$ \\
\hline
\end{tabular}

Los entrevistados concuerdan en su opinión, recordando que la empresa estima las entradas y salidas de dinero con la finalidad de agrupar el monto total que será destinado a un fondo para cancelar el IGTF. E1 inconveniente se genera después de implementada la técnica, entonces la respuesta sugiere que no, puesto que el fondo estimado no impide que se lleve a cabo la técnica, sin embargo, quien sí se ve afectada de cierto modo, es la administración del efectivo debido a que el dinero se mantiene inmóvil durante un tiempo, por lo que no se hace ningún movimiento para generar ganancias a la empresa, aunque es un apartado que resulta necesario porque a través de él se puede dar cumplimiento al IGTF.

Para sustentar lo anteriormente expuesto, Block y Hirt (2008) manifiestan que "la minimización de los saldos de efectivo, así como saber con precisión las fechas en que el efectivo se desplazará hacia adentro y hacia afuera de la compañía, mejora la rentabilidad general de una corporación”. (p. 125). Al identificar los deberes formales dentro del cumplimiento del Impuesto Grandes Transacciones Financieras que afectan la administración del efectivo en la empresa Vinccler, C.A, durante el período 2020.

Item 5: ¿Cómo maneja la empresa el deber formal de la Ley del IGTF que establece la obligación del suministro de información?

\begin{tabular}{l|l}
\hline \multicolumn{1}{c|}{ Entrevistado1 } & Entrevistado2 \\
\hline Se mantiene bajo los estándares de la ley. & $\begin{array}{l}\text { La empresa cumple con el deber formal, suministra la información requerida en la } \\
\text { Ley del IGTF y los demás deberes establecidos en el coT que guardan relación con } \\
\text { este. }\end{array}$
\end{tabular}

Los entrevistados coinciden al alegar que la empresa mantiene los estándares al cumplir con el pago del IGTF, esa es la forma correcta de cumplir con los deberes formales dentro de la misma. De esta manera se le da 
cumplimiento al artículo 19 de la Ley del Impuesto a las Grandes Transacciones Financieras, el cual expone que los sujetos pasivos del impuesto establecido en este Decreto con Rango, Valor y Fuerza de Ley, deben mantener y entregar a la Administración Tributaria Nacional, reportes detallados de las cuentas bancarias o contables, en los cuales se refleje el monto del impuesto pagado o retenido, si fuere el caso; ello sin perjuicio de los registros y demás procesos bancarios establecidos para el adecuado control del impuesto previsto en esta Ley.

Item 6: $\dot{\zeta} H a n$ incurrido en algún ilícito al momento de cumplir con la obligación del suministro de información sobre el IGTF? De ser asi, ¿Cuál ha sido la sanción?

Tabla 6. Respuestas del item 6

Entrevistado 1

Entrevistado 2

Los entrevistados reseñan que la empresa no ha incurrido nunca en algún ilícito al momento de cumplir con la obligación del suministro de información sobre el IGTF. Esto significa que la empresa cumple con todos los requisitos exigidos por la ley al momento de declarar su pago al Impuesto de las Grandes Transacciones Financieras. Lo encontrado coincide con lo propuesto por autores como Gomes (2011) quien es de la opinión de que el deber formal sirve de instrumento o garantía a la hora de que los jueces y tribunales enjuicien la conducta administrativa a través de su actividad de control.

Item 7: ¿Qué tipo de información maneja la empresa sobre las diversas sanciones que establece el COT a las cuales puede estar sujeto el contribuyente especial referente al IGTF?

Tabla 7. Respuestas del item 7

\begin{tabular}{l|l}
\multicolumn{2}{c}{ Entrevistado1 } \\
\hline $\begin{array}{l}\text { Se conocen con certeza los diferentes ilícitos formales establecidos en el COT y } \\
\text { por tanto se toman las debidas precauciones. Se nombraron distintos tipos de } \\
\text { faltas relacionadas al incumplimiento del IGTF que pueden recaer sobre el } \\
\text { contribuyente de este. }\end{array}$ & $\begin{array}{l}\text { El entrevistado maneja información completa con respecto a las distintas } \\
\text { sanciones que recaen sobre la empresa siendo contribuyente especial en el caso } \\
\text { de incumplir con sus deberes de acuerdo a la ley del IGTF. }\end{array}$ \\
&
\end{tabular}

De acuerdo con los entrevistados, están en pleno conocimiento acerca del tipo de información que debe manejar la empresa sobre las diversas sanciones que establece el COT y a las cuales puede estar sujeto el contribuyente especial referente al IGTF. Es decir, que se da pleno cumplimiento al artículo 108 del Código Orgánico Tributario, el cual afirma que el incumplimiento de cualquier otro deber formal sin sanción específica, establecido en las leyes y demás normas de carácter tributario, será sancionado con multa de cien unidades tributarias (100 U.T.); así como cuando los ilícitos formales previstos en este Capítulo sean cometidos por sujetos calificados como especiales por la Administración Tributaria, las sanciones pecuniarias aplicables serán 
aumentadas en un $200 \%$.

Item 8: ¿Cuáles de los hechos imponibles que realiza la empresa están sujetos al pago del IGTF?

Tabla 8. Respuestas del item 8

Entrevistado 1

Entrevistado 2

Débitos en cuentas bancarias y sesión de cheques.

Débitos en cuentas bancarias, la solicitud de chequeras, al momento de pagar la nómina se paga el IGTF solo por la comisión que cobra el banco para hacer la transacción, las transferencias en diferentes cuentas de la misma empresa pero a partir del segundo endoso, los cheques de gerencia en efectivo, sesión de cheques, y los pagos a nivel internacional.

Como puede observarse, la empresa paga el impuesto por las actividades económicas que realiza de acuerdo con los hechos imponibles que establece la Ley, o sea, que se le da cumplimiento al artículo 3 de la Ley del IGTF, el cual desglosa los elementos que constituyen hechos imponibles de este impuesto tal como se muestra a continuación:

Artículo 3. Constituyen hechos imponibles de este impuesto:

1. Los débitos en cuentas bancarias, de corresponsalía, depósitos en custodia o en cualquier otra clase de depósitos a la vista, fondos de activos líquidos, fiduciarios y en otros fondos del mercado financiero o en cualquier otro instrumento financiero, realizado en bancos y otras instituciones financieras.

2. La sesión de cheques, valores, depósitos en custodia pagados en efectivo y cualquier otro instrumento negociable, a partir del segundo endoso.

3. La adquisición de cheques de gerencia en efectivo.

4. Las operaciones activas efectuadas por bancos y otras instituciones financieras entre ellas mismas, y que tengan plazos no inferiores a dos (02) días hábiles bancarios.

5. La transferencia de valores en custodia entre distintos titulares, aun cuando no exista un desembolso a través de una cuenta.

6. La cancelación de deudas efectuadas sin mediación del sistema financiero, por el pago u otro medio de extinción.

7. Los débitos en cuentas que conformen sistemas de pagos organizados privados, no operados por el Banco Central de Venezuela y distintos del Sistema Nacional de Pagos.

8. Los débitos en cuentas para pagos transfronterizos.

Con esta información se evidencia que la empresa no tiene salidas de dinero adicional al correspondiente por los hechos imponibles establecidos y por consiguiente la administración del efectivo no se ve afectada por este elemento.

Item 9: ¿¿Desde la implementación del IGTF, cuál ha sido el comportamiento de las utilidades de la empresa? 
Tabla 9. Respuestas del item 9

Entrevistado 1

Entrevistado 2

Han disminuido las utilidades por motivo que el IGTF es una nueva salida de Disminución de las utilidades pues el impacto IGTF es una nueva salida de dinero.

dinero.

A1 85\% de los estudiantes del centro universitario expresó querer formar parte de los emprendedores que Los entrevistados opinan que la implementación del IGTF ha disminuido la entrada del efectivo a la empresa Vinccler, pues el mismo se ha convertido en una nueva salida de dinero, ello de una u otra manera afecta las utilidades obtenidas y a la liquidez de la empresa por ser una salida de dinero adicional por cada transacción realizada. Esto se debe, tal como lo plantea para Otálora (2009), a que el hecho imponible "es la causa que da origen al nacimiento de una obligación tributaria, es un hecho jurídico con relevancia económica”. (p. 133).

Item 10: ¿̇e ha visto afectada la empresa por el hecho de ser sujeto pasivo del IGTF? Justifique su respuesta.

Tabla 10. Respuestas del item 10

Entrevistado 1

Entrevistado 2

\begin{tabular}{|c|c|}
\hline Entrevistado 1 & Entrevistado 2 \\
\hline $\begin{array}{l}\text { Un poco en la parte financiera, sin embargo se ha planificado el adecuado pago } \\
\text { del IGTF. }\end{array}$ & $\begin{array}{l}\text { De cierto modo sí, porque es una responsabilidad más que recae sobre la } \\
\text { empresa, se debe dedicar tiempo, los empleados de esta área debemos estar } \\
\text { capacitados para no cometer errores que generen sanciones. }\end{array}$ \\
\hline
\end{tabular}

Los entrevistados opinan que la empresa sí se ha visto afectada por el hecho de ser sujeto pasivo del IGTF, debido a que se ha convertido en una responsabilidad para la cual hay que estar capacitado para evitar que el pago o cancelación de este impuesto se transforme en una sanción. De acuerdo a Acuña, Ruiz, Pedraz y García. (2007) "es sujeto pasivo el obligado tributario que, según la ley, debe cumplir la obligación tributaria principal, así como las obligaciones formales inherentes a la misma, sea como contribuyente o como sustituto del mismo". (p. 243). Por lo tanto, la empresa debe estar al día con este gravamen para evitar alguna sanción que pueda repercutir en la estabilidad financiera de la empresa.

Item 11: ¿La empresa ha disminuido sus movimientos mercantiles de modo de no exponer las bases imponibles al pago del IGTF? Justifique su respuesta.

Tabla 11. Respuestas del item 11

Entrevistado 1

Entrevistado 2 
Como puede observarse en este ítem, de acuerdo con la opinión de los entrevistados, la empresa se ha visto afectada con el pago del Impuesto en cuanto a su libertad de realizar la cantidad de transacciones financieras, esto debido a las magnitudes de dinero que representan las bases imponibles a las cuales se le aplicará el Impuesto. Vinccler ha llegado al punto de minimizar sus transacciones para evitar el exceso de dinero destinado al pago del Impuesto a las Grandes Transacciones Financieras, con la finalidad de no exponer de manera significativa las utilidades que al final de ejercicio económico disminuyen debido a las salidas adicionales de efectivo que representan el cumplir con el pago del impuesto.

La medida adoptada por la empresa es válida e incluso Ortega (2014) lo ratifica al decir "el sujeto se dedica a otra actividad menos gravada o exencionada en caso de los impuestos directos y disminuye el consumo de los artículos gravados o consume artículos menos gravados o exentos de gravamen en el caso de los impuestos directos". (p. 181).

Item No 12: ¿Vinccler se ha visto en la necesidad de aumentar los precios de sus servicios como medida de proteger sus utilidades, debido a que el IGTF no es deducible del ISLR? Justifique su respuesta.

Tabla 12. Respuestas del item 12

Entrevistado 1

Entrevistado2

$\mathrm{Si}$, han aumentados los precios para de cierto modo cubrir el pago del impuesto y no disminuir significativamente el nivel de utilidades también por los cambios de precios constantes en los insumos y en la nómina.
$\mathrm{Si}$, se ha tenido que incrementar los precios para modo cubrir el pago del impuesto, de esa forma se ha logrado no disminuir significativamente el nivel de utilidades, aunque esto también se debe a los cambios de precios constantes en los insumos.

Fuente: realización propia

Como se observa en las respuestas de los entrevistados, la empresa sí ha tenido que incrementar los precios de sus servicios como una medida para proteger sus utilidades, debido a que el IGTF no es deducible del ISLR.

Tal como lo revela el artículo 18 de la Ley del IGTF, cuando plantea que el impuesto previsto en este Decreto con Rango, Valor y Fuerza de Ley no será deducible del Impuesto Sobre la Renta. Esto de una u otra manera coincide con lo expuesto por Van Horne y Wachowicz (2010), quienes indican que casi todas las decisiones de negocios se ven afectadas directa o indirectamente por los impuestos. Mediante su poder impositivo, el gobierno federal, estatal y local tienen una influencia profunda en el comportamiento de los negocios y sus dueños.

\section{Conclusiones}

En relación a la examinación las técnicas de administración del efectivo que maneja la empresa Vinccler C.A., se evidenció que existe discrepancia en algunas respuestas, de igual manera, se obtuvo información de que la empresa adoptó una medida para administrar su dinero algo similar a la técnica del presupuesto de efectivo, de acuerdo a ello, se establece una similitud por cuanto los resultados obtenidos se asemejan a los que el presupuesto de efectivo le proporciona al interesado, es decir, se estiman los movimientos del dinero, conociéndose las entradas y salidas de este para, de ese modo, tomar las decisiones y medidas necesarias. 
Con esta medida la empresa ha visto beneficiada la administración de su efectivo, por considerar que es la manera acorde para cumplir con las responsabilidades del contribuyente del Impuesto a las Grandes Transacciones Financieras, sin embargo, existen otras técnicas de la administración del efectivo con las cuales Vinccler puede también percibir resultados positivos e incluso evitar ciertos inconvenientes al momento de implementarlas.

En cuanto a la identificación de los deberes formales dentro del cumplimiento del Impuesto a las Grandes Transacciones Financieras que afectan la administración del efectivo en la empresa Vinccler C.A. durante el período 2016, se dio pleno cumplimiento a los mismos en función de los artículos propuestos por la Ley del Impuesto a las Grandes Transacciones Financieras. Conociendo además, que se realizan los registros conforme a lo planteado en la legalidad venezolana para no incurrir en sanciones que les puedan ocasionar una mayor pérdida de dinero a la empresa.

En función de la caracterización de los elementos relacionados con el cumplimiento del Impuesto a las Grandes Transacciones Financieras que afectan la administración del efectivo en la empresa Vinccler C.A. durante el período 2020, se obtuvo la información de que en la empresa tienen pleno conocimiento de los elementos que los conducen a cumplir con el pago del Impuesto a las Grandes Transacciones Financiera. Los empleados del área administrativa manejan la información necesaria acerca del hecho imponible, así como de las consecuencias que les trae ser Sujetos Pasivos, de la Obligación del Pago del Impuesto, su Base Imponible y la No Deducibilidad del impuesto IGTF el cual no se utiliza como desgravamen en el pago del ILSR.

Finalmente, lo que respecta al análisis de la incidencia del Impuesto a las Grandes Transacciones Financieras en la empresa Vinccler, de acuerdo con la información recabada a través de la entrevista, se pudo conocer que la problemática encontrada al principio de la investigación en cuanto a la deficiencia en el manejo del dinero y todo lo que el cumplimiento del impuesto afecta la administración del efectivo, de cierto modo fue solventada actualmente con la implementación de la Técnica del Presupuesto de Efectivo, sin embargo, en las posteriores recomendaciones se mencionan otras técnicas reconocidas que también pueden ayudar al desenvolvimiento de la organización.

Por su parte, el cumplimiento de los deberes formales y el adecuado conocimiento respecto a los elementos del Impuesto a las Grandes Transacciones Financieras favorecen a la empresa, puesto que Vinccler no ha destinado dinero adicional como consecuencia de una mala gerencia en lo que el cumplimiento del Impuesto se refiere, evitándose de esta manera un impacto negativo para la administración del efectivo y al mismo tiempo eliminar una posible incidencia económica.

Se sugiere a la empresa implementar técnicas de la administración del efectivo adicional, la Técnica de los Estados Financieros Proyectados les permitirá obtener un mayor conocimiento acerca de los niveles futuros de efectivo, cuentas por pagar, cuentas por cobrar, las utilidades, entre otras cuentas. Sería de gran ayuda para la empresa debido a que el efectivo es el recurso más importante que tiene toda organización, por lo que su administración correcta es crucial para la adecuada y eficaz operatividad de la misma.

Otra técnica que pudiera emplear la empresa se corresponde al Modelo Miller- Orr por ser este un modelo que permite controlar la cantidad de dinero que se debe mantener disponible en efectivo dentro de la empresa, además, el mismo resulta oportuno cuando los flujos de efectivo son inciertos sabiendo que fluctúan día a día. Como es el caso que ocupa la investigación, puesto que los precios del mercado se encuentran en permanente fluctuación en lo que a las empresas constructoras se refiere. 
De igual modo se debe seguir de cerca los elementos del cumplimiento del Impuesto a las Grandes Transacciones financieras por si en algún momento llegaran a sufrir algún tipo de modificación en cuanto a los diferentes hechos imponibles o en cualquiera de los otros elementos la empresa tome las medidas necesarias para evitar errores que conlleven a posibles repercusiones económicas y un descontrol en la administración del efectivo.

\section{REFERENCIAS}

Acuña, J.; Ruiz, J.; Pedraz, M. y García, A. (2007). Procedimientos tributarios: Normas comunes gestión e inspección. España: Nueva imprenta, S.A.

Arias, F. (2006). El proyecto de investigación: Introducción a la metodología científica (6a ed.). Caracas: Episteme.

Block, S. y Hirt, G. (2008). Fundamentos de la Administración Financiera (12a ed.). México: Litografía Ingramex.

Constitución de la República Bolivariana de Venezuela. (1999).Gaceta Oficial de la República Bolivariana de Venezuela,5.908. Febrero 19, 2009.

Diccionario Básico Tributario Contable (2016). [Página web en línea].

Disponible en: http//www.eumed.net/cursecon/dic/dbtc/P.htm

Gomes, M. (2011). Instrumentos para la unificación de criterios administrativos en materia tributaria [Libro en línea]. Consultado el 5 de octubre de 2020 en: https://www.google.co.ve/?gfe_ rd=cr\&ei=Ffv3V5vcNK6yjgW38YXYCw\&gws_rd=ssl\#q=marcus+livio+gomes\&tbm=bks

Ley de Impuesto a las Grandes Transacciones Financieras. (Decreto No 2.169). (2015, diciembre 30). Gaceta Oficial de la República Bolivariana de Venezuela, 6.210 (Extraordinaria), Febrero 01, 2016.

Ley de Impuesto Sobre la Renta. (Decreto No 1.435). (2014, noviembre 17). Gaceta oficial de la República Bolivariana de Venezuela, 6.152 (Extraordinaria).

Noya, N., Fernández, N., Andrada, D., Gerez, L., González, D. y R ～icotta,N. (2014). Finanzas Públicas: Temas de Cátedra. Neuquén: Educo.

Ortega, A. (2014). Hacienda Pública: las Finanzas del Estado (5ta ed.) Bogotá: ECOE Ediciones.

Otálora, C. (2009). Economía fiscal. Bolivia: Plural editores

Rodríguez, L. (2004). El Efectivo y la Conciliación Bancaria. Mérida: Venezuela.

Van Horne, J. y Wachowiez, J. (2010). Fundamentos de la Administración Financiera (13a ed.) México: Pearson Educación de México, S.A. 


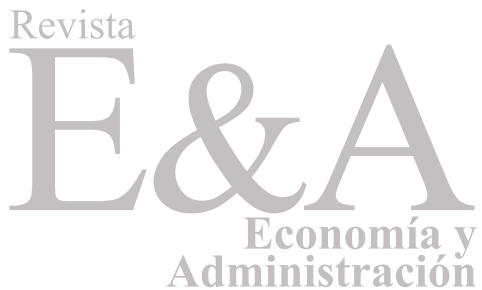

